\title{
Genome mining and biosynthesis of fumitremorgin-type alkaloids in ascomycetes
}

\author{
Shu-Ming Li \\ This review summarizes the recent progress on the biosynthesis of fumitremorgin-type alkaloids; that is, the identification of the \\ biosynthetic gene clusters from genome sequences by genome mining and proof of gene function by molecular biological and \\ biochemical investigations.
}

The Journal of Antibiotics (2011) 64, 45-49; doi:10.1038/ja.2010.128; published online 10 November 2010

Keywords: biosynthesis; fumitremorgin-type alkaloids; genome mining

\section{INTRODUCTION}

Indole alkaloids of the fumitremorgin-type contain tryprostatins, cyclotryprostatins, spirotryprostatins, fumitremorgins and verruculogen as well as derivatives thereof. These compounds have been isolated from various fungal sources, mainly from Aspergillus and Penicillium strains. They carry not only intriguing chemical structures, but also interesting biological and pharmacological activities. Early-feeding experiments showed that the members of this group of natural products are derived from tryptophan, proline and mevalonic acid. Recently, biosynthetic gene clusters of the fumitremorgin-type alkaloids consisting very likely of nine genes each has been identified in genomes of one Neosartorya fischeri and three Aspergillus fumigatus strains. The functions of seven genes and their roles in the biosynthesis were proven by molecular biological and biochemical approaches. The experimentally identified end product of the cluster is until now verruculogen.

\section{STRUCTURES OF FUMITREMORGIN-TYPE ALKALOIDS AND THEIR PRODUCERS AS WELL AS BIOLOGICAL AND PHARMACOLOGICAL ACTIVITIES}

Prenylated indole alkaloids are hybrid natural products containing indole/indoline and isoprenoid moieties or structures derived thereof. ${ }^{1,2}$ Fungal secondary metabolites derived from the diketopiperazine brevianamide $\mathrm{F}$ (1) consisting of L-tryptophan and Lproline (Figure 1) represent a large group within the prenylated indole alkaloids. ${ }^{1,2}$ The enormous structure diversity is only possible by prenylation and other modification reactions of the cyclic dipeptide skeleton. Derivatives of the regularly C2-prenylated brevianamide F; that is, tryprostatin B (2), are classified as fumitremorgin-type alkaloids, which include tryprostatins (2 and 3), spirotryprostatins (4 and 5), cyclotryprostatins (for example 6), fumitremorgins (7-10) and verruculogen (11). ${ }^{1,2}$ Finding and proof of the genetic information for the biosynthesis of these compounds are focus of this review. Reversely C2-prenylated brevianamide F; that is, deoxybrevianamide E (12), is the biosynthetic precursor of brevianamides; for example, brevianamide A (13), austamide (14) and notoamides like notoamide A (15).$^{2,3}$ During the reviewing process of this manuscript, a biosynthetic gene cluster for notoamides was identified in an Aspergillus sp. and two prenyltransferases from this cluster including NotF, a brevianamide F C2 reverse prenyltransferase, have been characterized biochemically. ${ }^{4}$

Simple fumitremorgin-type alkaloids with one prenyl moiety at position $\mathrm{C} 2$ are tryprostatins (2 and 3 ) or derivatives thereof. Spirotryprostatins (4 and 5) and fumitremorgin C (8) as well as its demethoxylated derivative $\mathbf{7}$ can be considered as derivatives of $\mathbf{2}$ and 3 after cyclization and modification. All of these compounds were isolated from several fungal strains. ${ }^{5-7}$ Some of these substances have been found to show interesting biological and pharmacological activities. For example, $\mathbf{2}$ and $\mathbf{3}$ as well as their diastereomeres were found to exhibit cytotoxicity toward various cancer cell lines, even more potent than etoposide. ${ }^{8,9}$ Compounds $\mathbf{8}$ and $\mathbf{3}$ are specific inhibitors of the breast cancer resistance protein, an ATP-binding cassette $(\mathrm{ABC})$ transporter (ABCG2) and can reverse the resistance of some tumor cell lines against antitumor agents. ${ }^{10-12}$ Therefore, 2, 3 and $\mathbf{8}$ as well as their analogues could serve as interesting candidates for development of anticancer agents.

The basis for the discovery of fumitremorgin-type alkaloids in the 1970 s was their ability to induce tremors in vertebrates. ${ }^{13,14}$ As results, fumitremorgins A (10) and B (9) as well as verruculogen (11) (Figure 1) were identified as tremorgenic mycotoxins. ${ }^{13-15}$ Verruculogen (11) containing an endoperoxide bridge between the two prenyl moieties of fumitremorgin B (9) was found in several strains of ascomycota ${ }^{16,17}$ including A. fumigatus ${ }^{5,18,19}$ and N. fischeri ${ }^{20}$ as well as Penicillium strains. ${ }^{21,22}$ Fumitremorgin A (10) carries, in 


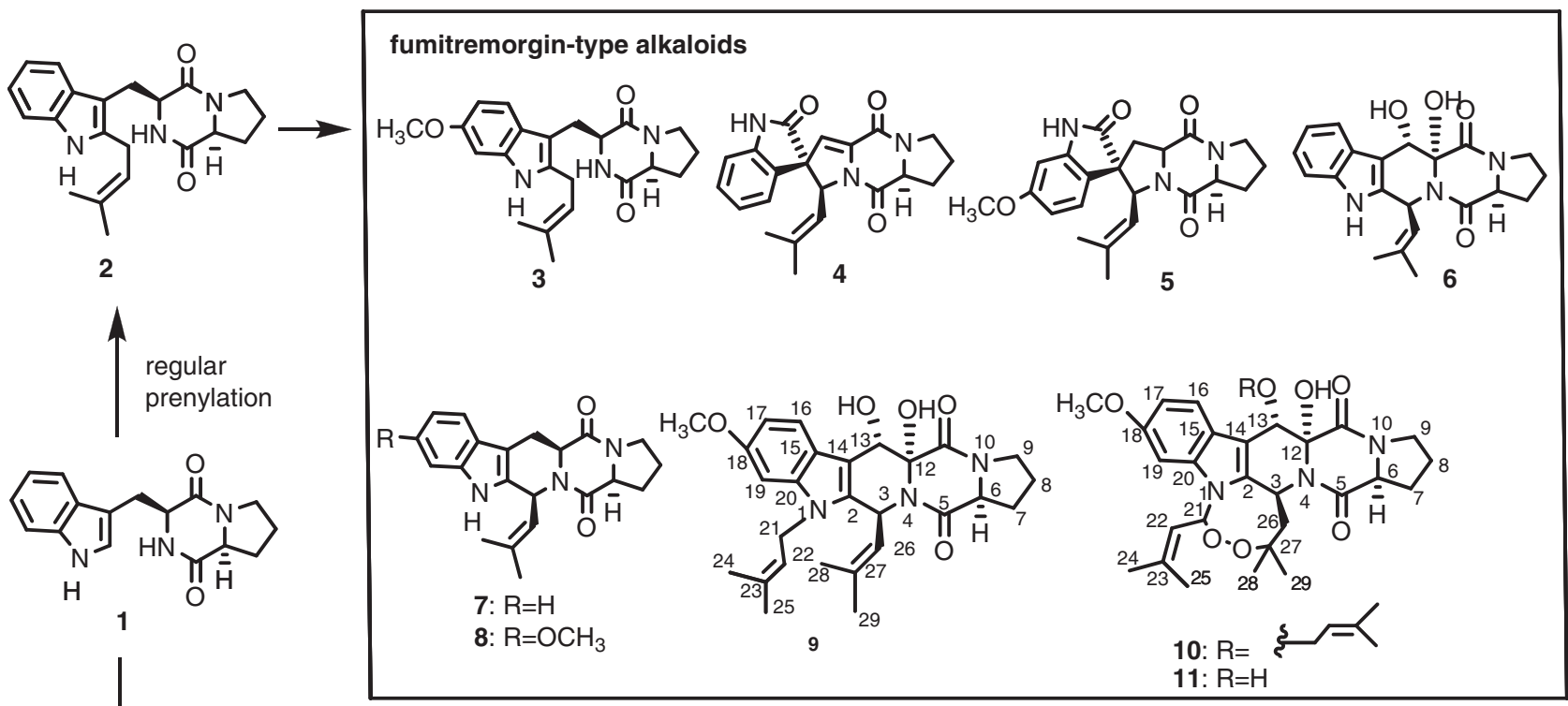

reverse

prenylation

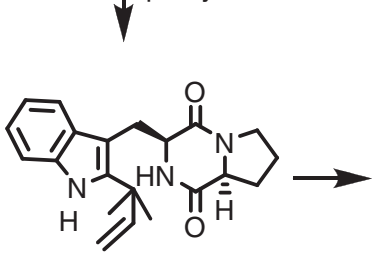

12

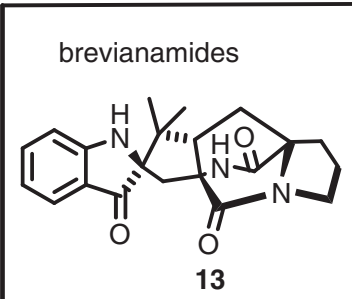

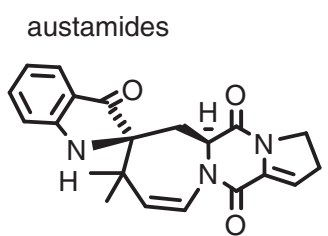

14

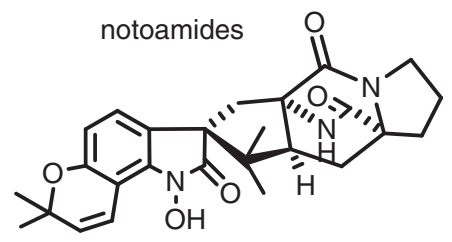

15

Figure 1 Structures of prenylated indole alkaloids derived from cyclo-L-tryptophan-L-proline and their biosynthetic relationship: brevianamide $\mathrm{F}$ (1), tryprostatins B (2) and A (3), spirotryprostatins B (4) and A (5), cyclotryprostatin C (6), demethoxyfumitremorgin C (7), fumitremorgin C (8), fumitremorgins $B(9)$ and $A(10)$, verruculogen (11), deoxybrevianamide $E(12)$, brevianamide $A(13)$, austamide (14) and notoamide $A(15)$.

comparison to verruculogen (11), an additional O-prenyl moiety at C13 and is produced by A. fumigatus and N. fischeri, ${ }^{19,20,23}$ but not by the genus of Penicillium. Verruculogen (11), associated with A. fumigatus hyphae and conidia, was reported to be able to modify the electrophysiological properties of human nasal epithelial cells and could be involved in the pathogenesis of this fungus. ${ }^{24}$

Recently, a number of modified derivatives of 2-11 have been isolated from Aspergillus strains including A. fumigatus. ${ }^{18,25}$ Some of them differ from 2-11 just in their stereochemistry at C12 and C13; that is, $\beta$-hydroxyl instead of $\alpha$-hydroxyl groups. In some cases, the 13-hydroxyl was oxidized to keto group or methylated to methoxy group. Compounds with modifications at one or both prenyl moieties, such as hydroxylation, oxidation and peroxidation, have also been isolated. ${ }^{18,25}$

\section{BIOSYNTHETIC STUDIES ON FUMITREMORGIN-TYPE ALKALOIDS BY FEEDING EXPERIMENTS}

Feeding experiments with ${ }^{13} \mathrm{C}$ or ${ }^{14} \mathrm{C}$-labeled acetate, proline, mevalonic acid and methionine in Aspergillus and Penicillium strains, and characterization of the isolated secondary metabolites such as fumitremorgin $\mathrm{B}$ and verruculogen revealed clearly that the common precursor of these compounds is the cyclic dipeptide brevianamide F consisting of L-tryptophan and L-proline. The prenyl moieties are derived from mevalonic acid. Cultivation of Penicillium verruculosum under an atmosphere of ${ }^{18} \mathrm{O}_{2}$ and characterization of verruculogen indicated the formation of the endoperoxide bond by incorporation of an oxygen molecule. ${ }^{26}$ For details on the results of the feeding experiments, the review by Professor Williams ${ }^{2}$ and literature cited there are recommended.

\section{IDENTIFICATION OF THE BIOSYNTHETIC GENE CLUSTERS OF} FUMITREMORGIN-TYPE ALKALOIDS BY GENOME MINING

Significant progress on the biosynthesis of fumitremorgin-type alkaloids was only achieved after availability of genome sequences. A gene cluster for the biosynthesis of fumitremorgin-type alkaloids was initially identified by our group in the genome of A. fumigatus Af293. ${ }^{27}$ This strain was the first sequenced Aspergillus species and its genome sequence was released to the public in 2003. By blasting the genome sequence with $c p d 1$, a prenyltransferase gene coding for a 4-dimethylallyltryptophan synthase in the biosynthesis of ergot alkaloids in Claviceps purpurea, ${ }^{28}$ a segment of $25 \mathrm{kbp}$ on chromosome 8 was identified as a putative biosynthetic gene cluster for fumitremorgin B. ${ }^{27}$ The identified cluster was proposed to comprise 10 genes. ${ }^{27}$ Later on, orthologous clusters were also identified in A. fumigatus A1163 and N. fischeri NRRL181. ${ }^{29,30}$ Comparison of these clusters revealed the presence of all of the 10 genes in both strains. Remarkably, the orthologous gene of orf $17^{27}$ in NRRL181, termed $f t m P$ in the $\mathrm{PhD}$ thesis of A Grundmann, ${ }^{30}$ is separated from $\mathrm{ftmO}$ by a segment of $24 \mathrm{kbp}$ and is likely not a member of the gene cluster of fumitremorgintype alkaloids. ${ }^{30}$ Therefore, we speculate that the mentioned clusters consist of nine genes each as illustrated in Figure 2, which was also identified in A. fumigatus BM939 (Figure 2). ${ }^{31}$ However, it cannot be excluded that different strains carry similar, but not identical clusters 


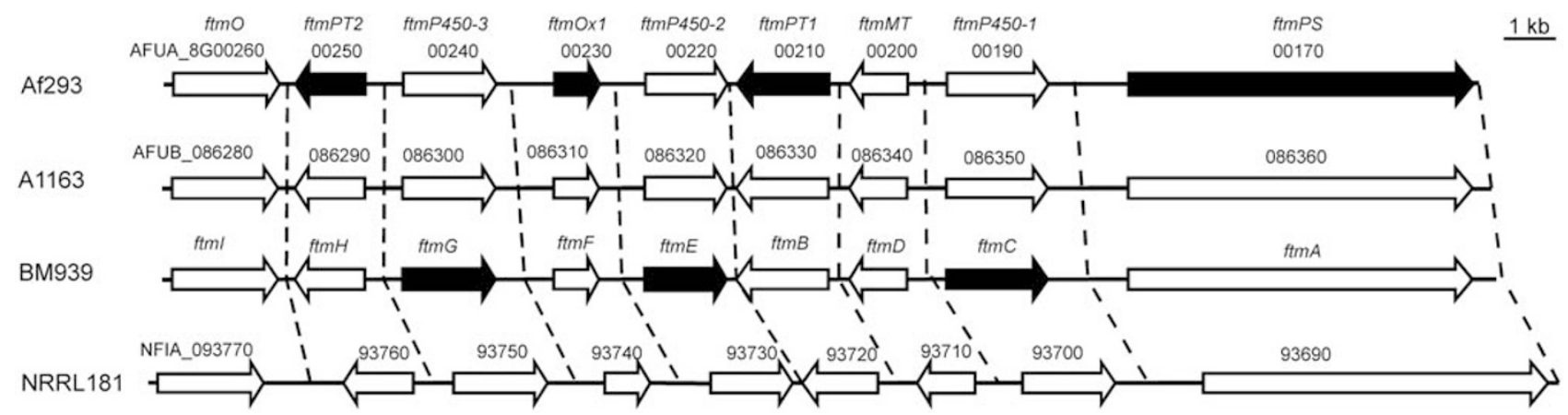

Figure 2 Biosynthetic gene clusters of fumitremorgin-type alkaloids in Aspergillus fumigatus Af293, A1163, BM939 and Neosartorya fischeri NRRL181. Orthologous genes are indicated between dotted lines. Functions of genes with black arrows have been proven experimentally.

and produce different end products. The nine genes from the four strains share high homology to each other with sequence identities of 85-99\% on the amino acid level. Furthermore, they have same order and orientation in these clusters. The end product of this cluster was proven to be verruculogen (11) (see below), ${ }^{32}$ but it could not be excluded that fumitremorgin A (10) is the true end product instead. The fumitremorgin/verruculogen cluster was very likely not expressed in A. fumigatus Af293; therefore, no secondary metabolites of this cluster could be detected in this strain. ${ }^{33}$ In contrast, a number of intermediates including tryprostatins $\mathrm{A}(3)$ and $\mathrm{B}(2)$, fumitremorgin C (8), 12,13-dihydroxyfumitremorgin C, fumitremorgin B (9) as well as verruculogen (11) were isolated from A. fumigatus BM939..$^{5}$ No data on the expression of the gene clusters in A. fumigatus A1163 and N. fischeri NRRL181 are available.

\section{MOLECULAR BIOLOGICAL AND BIOCHEMICAL INVESTIGATIONS ON THE BIOSYNTHESIS OF FUMITREMORGIN-TYPE ALKALOIDS}

Functional proof of the biosynthetic genes and gene cluster of fumitremorgin-type alkaloids was carried out by gene inactivation experiments in the producing strain BM939, ${ }^{31}$ by identification of the accumulated secondary metabolites after heterologous expression in Aspergillus strains ${ }^{33,34}$ or by biochemical investigation of the recombinant enzymes from Escherichia coli or Saccharomyces cerevisiae. ${ }^{27,31,32,35}$ In total, functions of seven genes have been proven experimentally. On the basis of the obtained bioinformatic, molecular biological and biochemical results, the following biosynthetic pathway has been postulated (Figure 3). ${ }^{1}$

The biosynthesis of fumitremorgin-type alkaloids begins by condensation of the two amino acids L-tryptophan and L-proline to brevianamide $\mathrm{F}$, catalyzed by the non-ribosomal peptide synthetase FtmPS (also termed FtmA), which was proven by heterologous overexpression of the NRPS gene ftmPS in Aspergillus and identification of the accumulated product brevianamide $\mathrm{F}^{33}$ Brevianamide $\mathrm{F}$ is then prenylated by the prenyltransferase FtmPT1/FtmB in the presence of dimethylallyl diphosphate, resulting in the formation of tryprostatin $\mathrm{B}$, as demonstrated by using the recombinant FtmPT1. ${ }^{27}$ By gene inactivation in A. fumigatus BM939 and heterologous expression in S. cerevisiae, Kato et al. ${ }^{31}$ showed that the three cytochrome $\mathrm{P} 450$ enzymes, FtmP450-1/FtmC, FtmP450-2/FtmE and FtmP450-3/FtmG, are responsible for the conversion of tryprostatin $\mathrm{B}$ (2) to 6-hydroxytryprostatin B, tryprostatin A (3) to fumitremorgin C (8) and fumitremorgin $\mathrm{C}(\mathbf{8})$ to 12,13-dihydroxyfumitremorgin $\mathrm{C}$, respectively. Using recombinant enzymes, we have demonstrated that FtmPT2/FtmH catalyzes the prenylation of 12,13-dihydroxyfumitre- morgin $\mathrm{C}$ in the presence of dimethylallyl diphosphate, resulting in the formation of fumitremorgin $\mathrm{B},{ }^{35}$ which is then converted to verruculogen by FtmOx1/FtmF by inserting an endoperoxide bond between the two prenyl moieties. ${ }^{32}$ We have shown that FtmOx1 reaction was absolutely dependent on the presence of $\mathrm{Fe}(\mathrm{II})$ and $\alpha$-ketoglutarate, and therefore functions as a non-heme $\mathrm{Fe}(\mathrm{II})$ and $\alpha$-ketoglutaratedependent dioxygenase. Known $\alpha$-ketoglutarate-dependent dioxygenases usually transfer one oxygen atom to the substrate and another to $\alpha$-ketoglutarate resulting in the formation of succinate. ${ }^{36}$ In contrast, both oxygen atoms from a single $\mathrm{O}_{2}$ molecule were incorporated into the structure of verruculogen, which was proven by incubation of fumitremorgin $\mathrm{B}$ with $\mathrm{FtmOx} 1$ in ${ }^{18} \mathrm{O}_{2}$ enriched atmosphere. ${ }^{32}$ Therefore, FtmOxl represents the first endoperoxide forming non-heme $\mathrm{Fe}(\mathrm{II})$ and $\alpha$-ketoglutarate-dependent dioxygenase reported so far. Until now, functions of two genes $\mathrm{ftmMT} / \mathrm{ftmD}$ and $\mathrm{ftmO} / \mathrm{ftmI}$ have to be proven experimentally. The putative methyltransferase FtmMT/FtmD is expected for the conversion of 6-hydroxytryprostatin B to tryprostatin A. ${ }^{27}$ Kato et al. ${ }^{31}$ reported that inactivation of $\mathrm{ftmO} / \mathrm{ftmI}$ in A. fumigatus BM939 did not abolish the fumitremorgin $B$ accumulation. Using overproduced and purified $\mathrm{His}_{6}$-FtmO, we could not provide any evidence on its role in the biosynthesis of fumitremorgin-type alkaloids (unpublished results).

Until now, a conversion of verruculogen to fumitremorgin A was demonstrated neither by an overproduced protein nor by a crude protein extract from a producer. Furthermore, no candidate gene could be identified in the clusters mentioned above. Verruculogen was found in much more strains including A. fumigatus, ${ }^{5,18,19}$ N. fischeri $^{20,37}$ and Penicillium species ${ }^{21,22}$ than fumitremorgin A, which was not identified in Penicillium strains. This indicates a genetic difference of these strains or non-expression of the prenyltransferase being responsible for the transfer of a prenyl moiety to the hydroxyl group of verruculogen in Penicillium strains.

As mentioned previously, a series of derivatives of the intermediates in the biosynthesis illustrated in Figure 3, including stereoisomers and oxidation products, have been isolated from different Aspergillus strains. ${ }^{7,18,25,38}$ Obviously, the nine genes of the clusters mentioned above could not account for the synthesis of all of these compounds. It should be questioned whether some of these compounds are enzymatic products in living organisms or results of chemical reactions; for example, oxidation by atmospheric oxygen. It cannot be excluded that unspecific genes/enzymes out of the clusters convert the biosynthetic intermediates to the respective products. It is of course also possible that the biosynthetic enzymes in the cluster catalyze similar reactions, resulting in the formation of side products of the pathway. The last possibility is that there are more than nine genes in the related clusters in such strains. 

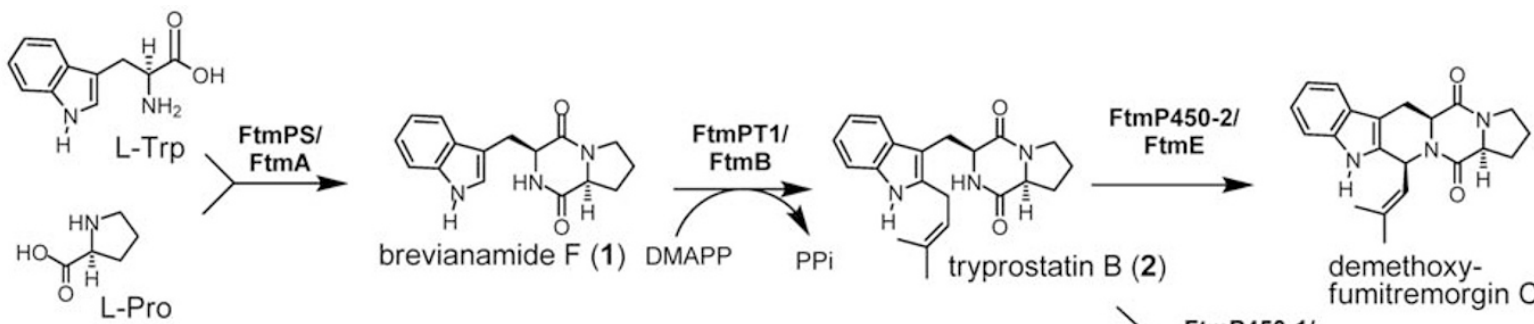

demethoxyfumitremorgin $\mathrm{C}(7)$
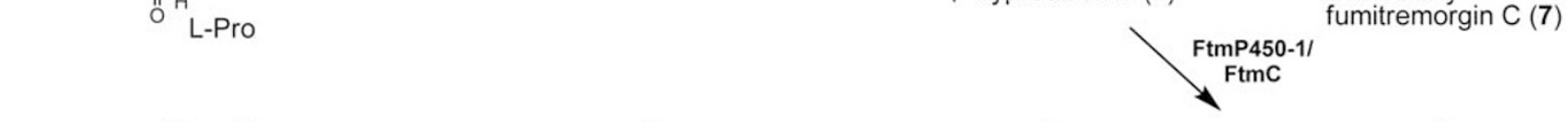<smiles>CC=CC1c2[nH]c3cc(OC)ccc3c2[C@@H](O)[C@@]2(O)C(=O)N3CCC[C@H]3C(=O)N12</smiles>

12,13-dihydroxyfumitremorgin C

fumitremorgin B (9)

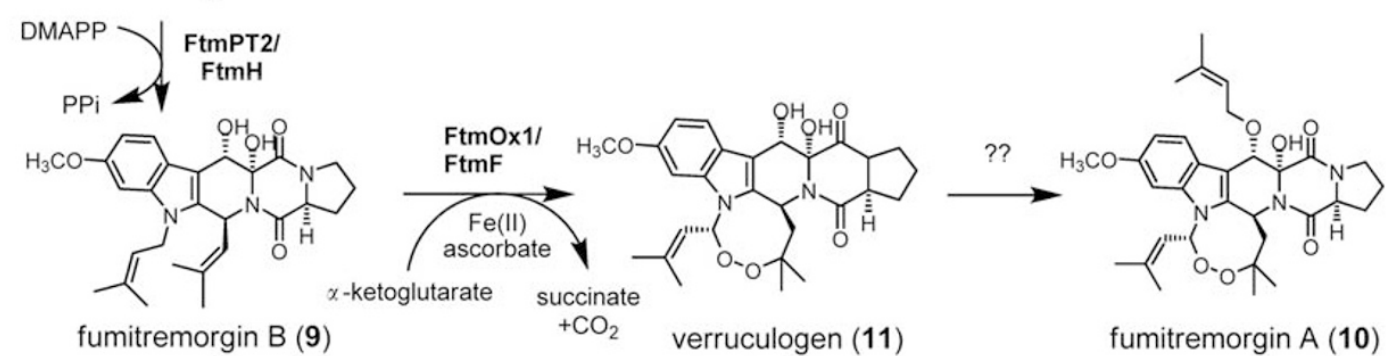<smiles>COc1ccc2c3c([nH]c2c1)C(C=C(C)C)N1C(=O)[C@@H]2CCCN2C(=O)C1Cc1c-3[nH]c2cc(C(F)(F)F)ccc12</smiles>

fumitremorgin $\mathrm{C}(8)$

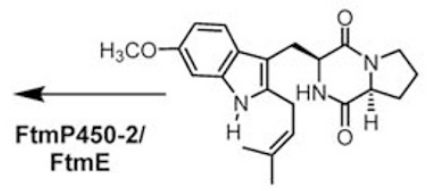

tryprostatin A (3)

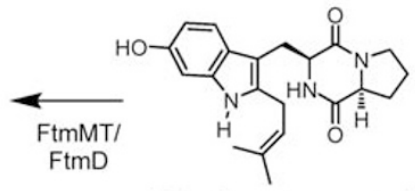

6-hydroxytryprostatin B

Figure 3 Proposed biosynthetic pathway for verruculogen and fumitremorgin A in Aspergillus fumigatus. Abbreviation: DMAPP, dimethylallyl diphosphate; $\mathrm{PPi}$, pyrophosphate.

\section{CONCLUSION AND DISCUSSION}

Since 1970's, a number of fumitremorgin-type alkaloids and related substances have been identified in various fungal strains. ${ }^{2}$ Significant progress on the biosynthesis of these compounds was only achieved after sequence release of genome sequencing projects, especially for Aspergillus strains in 2003/2004. With an exception for the methyltransferase, the genes/enzymes involved in the reaction steps from L-tryptophan and L-proline to verruculogen have been characterized experimentally. This provides an excellent example for identification of genetic information of natural products by genome mining. The availability of more and more genome sequences provides an enormous chance for the study of secondary metabolites. Functional proof of these genes and identification of the natural products coded by the gene clusters would be the main challenges for natural product chemists and biologists in the next years or even decades.

\section{ACKNOWLEDGEMENTS}

The works in the author's laboratory were supported by grants from the Deutsche Forschungsgemeinschaft (Li844/1-3).

$1 \mathrm{Li}, \mathrm{S} .-\mathrm{M}$. Prenylated indole derivatives from fungi: structure diversity, biological activities, biosynthesis and chemoenzymatic synthesis. Nat. Prod. Rep. 27, 57-78 (2010).

2 Williams, R. M., Stocking, E. M. \& Sanz-Cervera, J. F. Biosynthesis of prenylated alkaloids derived from tryptophan. Topics Curr. Chem. 209, 97-173 (2000).

3 Kato, H. et al. Notoamides A-D: prenylated indole alkaloids isolated from a marinederived fungus, Aspergillus sp. Angew. Chem. Int. Ed. Engl. 46, 2254-2256 (2007).

4 Ding, Y. et al. Genome-based characterization of two prenylation steps in the assembly of the stephacidin and notoamide anticancer agents in a marine-derived Aspergillus sp. J. Am. Chem. Soc. 132, 12733-12740 (2010).
5 Cui, C. B., Kakeya, H. \& Osada, H. Novel mammalian cell cycle inhibitors, tryprostatins A, B and other diketopiperazines produced by Aspergillus fumigatus. II. Physicochemical properties and structures. J. Antibiot. 49, 534-540 (1996).

6 Cui, C. B., Kakeya, H. \& Osada, H. Novel mammalian cell cycle inhibitors, spirotryprostatins $A$ and B, produced by Aspergillus fumigatus, which inhibit mammalian cell cycle at G2/M phase. Tetrahedron 52, 12651-12666 (1996).

7 Cui, C. B., Kakeya, H. \& Osada, H. Novel mammalian cell cycle inhibitors, cyclotryprostatins A-D, produced by Aspergillus fumigatus, which inhibit mammalian cell cycle at G2/M phase. Tetrahedron 53, 59-72 (1997).

8 Kondoh, M., Usui, T., Mayumi, T. \& Osada, H. Effects of tryprostatin derivatives on microtubule assembly in vitro and in situ. J. Antibiot. 51, 801-804 (1998).

9 Zhao, S. et al. Biological activity of the tryprostatins and their diastereomers on human carcinoma cell lines. J. Med. Chem. 45, 1559-1562 (2002).

10 Rabindran, S. K., Ross, D. D., Doyle, L. A., Yang, W. \& Greenberger, L. M. Fumitremorgin $C$ reverses multidrug resistance in cells transfected with the breast cancer resistance protein. Cancer Res. 60, 47-50 (2000).

11 Allen, J. D. et al. Potent and specific inhibition of the breast cancer resistance protein multidrug transporter in vitro and in mouse intestine by a novel analogue of fumitremorgin C. Mol. Cancer Ther. 1, 417-425 (2002).

12 Woehlecke, H., Osada, H., Herrmann, A. \& Lage, H. Reversal of breast cancer resistance protein-mediated drug resistance by tryprostatin A. Int. J. Cancer 107, 721-728 (2003).

13 Cole, R. J. et al. Tremorgenic toxin from Penicillium verruculosum. Appl. Microbiol. 24, 248-250 (1972).

14 Yamazaki, M., Sasago, K. \& Miyaki, K. Structure of fumitremorgin B (FTB), a tremorgenic toxin from Aspergillus fumigatus. J. Chem. Soc. Chem. Commun. 408-409 (1974).

15 Yamazaki, M., Fujimoto, H. \& Kawasaki, T. Structure of a tremorgenic metabolite from Aspergillus fumigatus, fumitremorgin A. Tetrahedron Lett. 1241-1244 (1975).

16 Steyn, P. S., Vleggaar, R. \& Rabie, C. J. Alkaloids from Aspergillus caespitosus. Phytochemistry 20, 538-539 (1981).

17 Sabater-Vilar, M., Maas, R. F., De Bosschere, H., Ducatelle, R. \& Fink-Gremmels, J. Patulin produced by an Aspergillus clavatus isolated from feed containing malting residues associated with a lethal neurotoxicosis in cattle. Mycopathologia 158, 419-426 (2004).

18 Wang, F. et al. Seven new prenylated indole diketopiperazine alkaloids from holothurian-derived fungus Aspergillus fumigatus. Tetrahedron 64, 7986-7991 (2008).

19 Horie, Y. \& Yamazaki, M. Productivity of tremorgenic mycotoxins, fumitremorgins A and B in Aspergillus fumigatus and allied species. Trans. Mycol. Soc. Jpn. 22, 113-119 (1981). 
20 Nielsen, P. V., Beuchat, L. R. \& Frisvad, J. C. Growth of and fumitremorgin production by Neosartorya fischeri as affected by temperature, light, and water activity. Appl. Environ. Microbiol. 54, 1504-1510 (1988).

21 Vleggaar, R., Horak, R. M. \& Maharaj, V. J. Stereochemical course of ring formation in fumitremorgin $B$ and verruculogen, metabolites of Penicillium verruculosum: investigation into the loss of stereochemical integrity of the geminal methyl groups. J. Chem. Soc. Chem. Commun. 274-275 (1993).

22 Gallagher, R. T. \& Latch, G. C. M. Production of the tremorgenic mycotoxins verruculogen and fumitremorgin B by Penicillium piscarium Westling. Appl. Envir. Microbiol. 33, 730-731 (1977).

23 Yamazaki, M., Fujimoto, H. \& Kawasaki, T. Chemistry of tremorogenic metabolites. I. Fumitremorgin A from Aspergillus fumigatus. Chem. Pharm. Bull. 28, 245-254 (1980).

24 Khoufache, K. et al. Verruculogen associated with Aspergillus fumigatus hyphae and conidia modifies the electrophysiological properties of human nasal epithelial cells. BMC. Microbiol. 7, 5 (2007).

25 Zhang, M. et al. Cytotoxic alkaloids and antibiotic nordammarane triterpenoids from the marine-derived fungus Aspergillus sydowi. J. Nat. Prod. 71, 985-989 (2008).

26 Horak, R. M. \& Vleggaar, R. Biosynthesis of verruculogen, a tremorgenic metabolite of Penicillium verruculosum: sterochemical course of peroxide ring formation. J. Chem. Soc. Chem. Commun. 1568-1570 (1987).

27 Grundmann, A. \& Li, S.- M. Overproduction, purification and characterization of FtmPT1, a brevianamide F prenyltransferase from Aspergillus fumigatus. Microbiology 151, 2199-2207 (2005).

28 Tudzynski, P. et al. Evidence for an ergot alkaloid gene cluster in Claviceps purpurea. Mol. Gen. Genet. 261, 133-141 (1999).
29 Steffan, N., Grundmann, A., Yin, W.- B., Kremer, A. \& Li, S.- M. Indole prenyltransferases from fungi: a new enzyme group with high potential for the production of prenylated indole derivatives. Curr. Med. Chem. 16, 218-231 (2009).

30 Grundmann, A. Molecular biological and biochemical investigations on the biosynthesis of mycotoxins from Aspergillus fumigatus. PhD Thesis, Heinrich-Heine-Univ. Düsseldorf (2008).

31 Kato, N. et al. Identification of cytochrome P450s required for fumitremorgin biosynthesis in Aspergillus fumigatus. Chembiochem 10, 920-928 (2009).

32 Steffan, N., Grundmann, A., Afiyatullov, A., Ruan, H. \& Li, S.- M. Ftm0x1, a non heme $\mathrm{Fe}(\mathrm{II})$ and alpha-ketoglutarate-dependent dioxygenase, catalyses the endoperoxide formation of verruculogen in Aspergillus fumigatus. Org. Biomol. Chem. 7, 4082-4087 (2009).

33 Maiya, S., Grundmann, A., Li, S.- M. \& Turner, G. The fumitremorgin gene cluster of Aspergillus fumigatus: identification of a gene encoding brevianamide $\mathrm{F}$ synthetase. Chembiochem 7, 1062-1069 (2006).

34 Maiya, S., Grundmann, A., Li, S.- M. \& Turner, G. Improved tryprostatin B production by heterologous gene expression in Aspergillus nidulans. Fungal Genet. Biol. 46, 436440 (2009).

35 Grundmann, A., Kuznetsova, T., Afiyatullov, S. S. \& Li, S.- M. FtmPT2, an Nprenyltransferase from Aspergillus fumigatus, catalyses the last step in the biosynthesis of fumitremorgin B. Chembiochem 9, 2059-2063 (2008).

36 Hausinger, R. P. Fe(II)/alpha-ketoglutarate-dependent hydroxylases and related enzymes. Crit. Rev. Biochem. Mol. Biol. 39, 21-68 (2004).

37 Larsen, T. 0. et al. Production of mycotoxins by Aspergillus lentulus and other medically important and closely related species in section Fumigati. Med. Mycol. 45, 225-232 (2007).

38 Cui, C. B., Kakeya, H. \& Osada, H. Spirotryprostatin B, a novel mammalian cell cycle inhibitor produced by Aspergillus fumigatus. J. Antibiot. 49, 832-835 (1996). 